\title{
The Microbiome Colorectal Cancer Puzzle: Initiator, Propagator, and Avenue for Treatment and Research
}

\author{
Reece J. Knippel, PhD, ${ }^{1}$ and Cynthia L. Sears, MD ${ }^{1,2,3}$
}

\begin{abstract}
The human gut microbiome has an ever-increasing role in the instigation and progression of colorectal cancer (CRC). Recent investigations have focused on identifying the key causative bacterial species and the composition and structure of the microbiome as a whole that ultimately lead to tumorigenesis in the colon. Understanding the bacterial mechanisms that promote CRC provides a rich area for the development of new screening modalities and therapeutics that may improve patient outcomes. This article reviews the various mechanisms that bacteria in the gut use to induce and/or promote tumor formation, discusses the application of the microbiome in the prevention and therapy of CRC, and provides directions for future research endeavors aiming to develop a more complete understanding of this complex phenomenon.
\end{abstract}

J Natl Compr Canc Netw 2021;19(8)986-992 doi: 10.6004/jnccn.2021.7062

\section{Background}

Colorectal cancer (CRC) persists as a global public health burden, as the second highest diagnosed cancer in women and men and the third highest cause of cancer-related deaths worldwide. ${ }^{1}$ The onset of tumor formation is multifactorial, consisting of a series of mutations and epigenetic changes over time, leading to the induction of oncogenes and suppression of tumor suppressor genes in the large bowel. ${ }^{1,2}$ Although inherited genetic predisposition syndromes, such as Lynch syndrome and familial adenomatous polyposis (FAP), are well described, they contribute to a minority of CRC cases. ${ }^{1}$ Most CRC cases arise sporadically as a direct result of environmental stimuli. ${ }^{1}$ Factors including obesity, smoking, alcohol consumption, a Western diet, and diabetes have been associated with risk for developing CRC. ${ }^{1}$ However, each of the CRC risk factors modifies the gut microbiome, the trillions of microbial species that reside within the colon. Thus, the gut microbiome has become an area of intense focus in the initiation and progression of carcinogenesis in the past decade. ${ }^{1,3}$ In this review, we discuss the mechanisms the microbiota likely use to lead to the development of CRC, applications of the microbiome in the treatment and diagnosis of disease, and directions for future research that may lead to the reduction of the global health burden of CRC.

\section{Microbial Mechanisms of Tumorigenesis}

The human gut microbiome consists of bacteria, archaea, fungi, and viruses, and a burgeoning literature has begun to link gut microbiome composition to a multitude of diseases ranging from schizophrenia to inflammatory bowel disease. ${ }^{4,5}$ Bacteria are highly abundant in the microbiome, estimated to be approximately $3.8 \times 10^{13}$ in total, ${ }^{6}$ and are the focus of this review. There are 3 primary ways by which bacteria foster the initiation and/or progression of tumor formation: (1) secretion of microbial products, including toxigenic molecules and reactive oxidative stress (ROS)-producing molecules and metabolites; (2) direct contact and interaction with host cells through attachment, invasion, 
and translocation; and (3) modification of the host genome and signaling pathways (Figure 1).

\section{Individual Bacteria Associated With CRC}

Theories regarding the temporal aspects of bacteriainduced CRC include the driver-passenger model and the hit-and-run model. In the first, one or more driver procarcinogenic bacteria work with collaborating microbiome partners, or passengers, to foster colon tumorigenesis; in the second, only transient colonization by a procarcinogenic bacterium is necessary and sufficient to drive tumorigenesis. Evidence exists for both models. ${ }^{7}$ Although a defined sequence of bacteria-driven events conclusively inducing CRC remains elusive, specific bacteria have been linked to human CRC. An overabundance of the gram-negative anaerobic oral commensal Fusobacterium nucleatum in stool and tissue has been identified as a potential CRC biomarker. ${ }^{8}$ Interestingly, $F$ nucleatum does not encode any as yet known toxins and very few canonical virulence factors. ${ }^{8}$ However, a series of experimental findings suggest that $F$ nucleatum (or, more specifically, 1 of its 4 subspecies) impacts colon carcinogenesis. These findings include that (1) the F nucleatum virulence factor FadA binds to the extracellular domain of E-cadherin, accelerating colon cancer cell proliferation through activation of host colonic epithelial cell (CEC) Wnt $/ \beta$-catenin signaling and Toll-like receptor 4 -activated signaling to nuclear factor- $\kappa \mathrm{B}(\mathrm{NF}-\kappa \mathrm{B})^{9}$ (notably, FadA gene expression in human CRC tissue is significantly upregulated compared with healthy tissue controls ${ }^{9}$ ); and (2) Fnucleatuminduces a proinflammatory myeloid-dominant tumor-promoting microenvironment while diminishing antitumor immunity by interfering

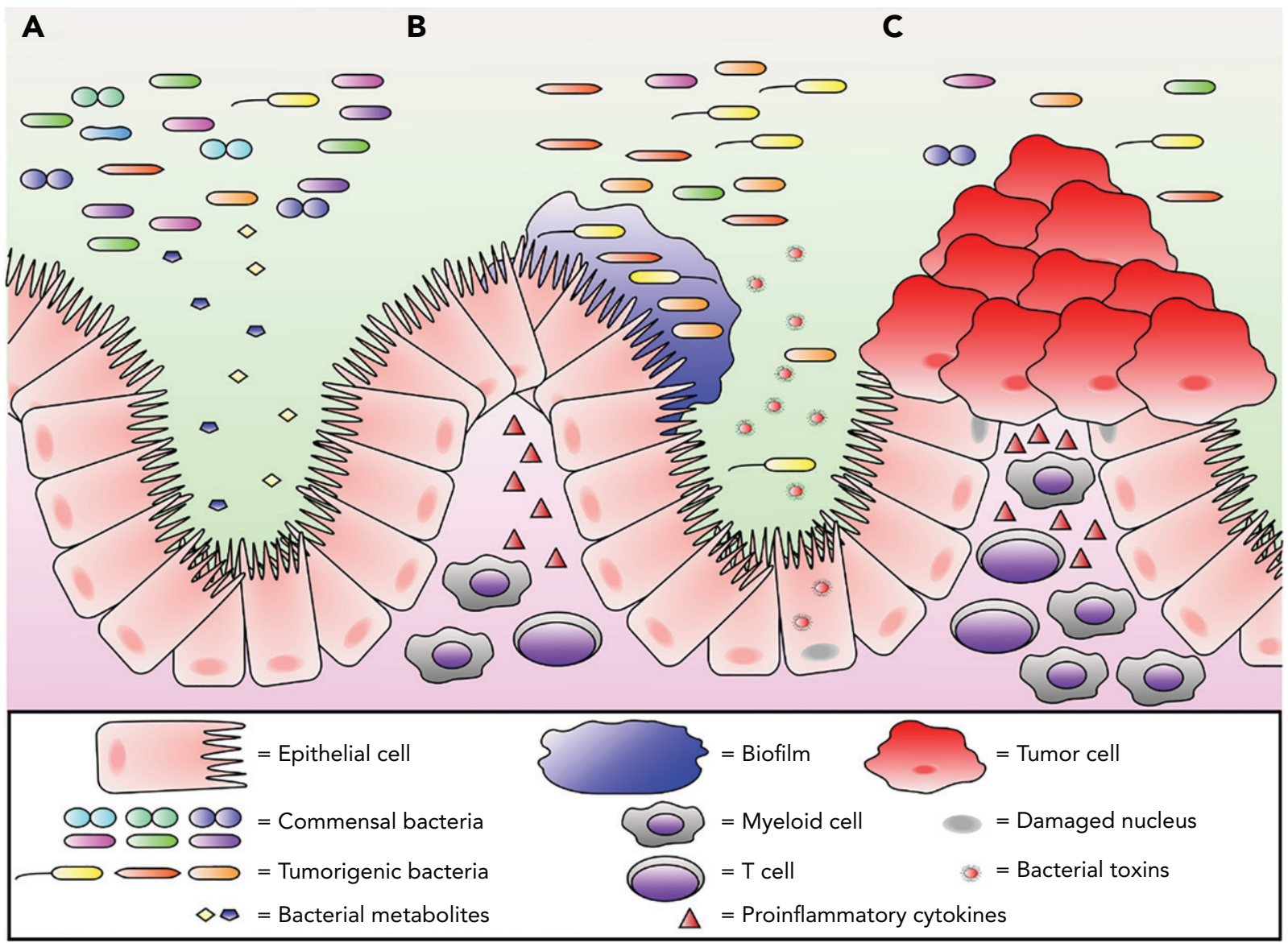

Figure 1. Microbial initiation and progression of tumorigenesis in the colon. (A) A microbiome with high diversity produces metabolites that regulate CEC and mucosal immune function in a primarily positive manner. (B) During dysbiosis, tumorigenic bacteria bloom and induce production of proinflammatory cytokines and recruitment of myeloid and T cells. Tumorigenic bacteria can also penetrate into the colon inner mucus layer to form biofilms on the surface of CECs that modulate host signaling pathways. Certain tumorigenic bacteria produce toxins, such as Bacteroides fragilis toxin and colibactin, that alter CEC signaling and/or damage CEC DNA, resulting in mutations and/or cytotoxicity that further alter host and microbial community function. (C) This multifactorial assault over time leads to clonal expansion of a CEC bearing one or more oncogenic mutations to yield colon neoplasia.

Abbreviation: CEC, colonic epithelial cell. 
with recruitment of tumor-infiltrating lymphocytes and activation of the TIGIT receptor, an immune checkpoint molecule, on natural killer cells. ${ }^{8,10}$ Despite the rapid expansion of literature on the tumorigenic aspects of F nucleatum, the causative role of this bacterium in CRC remains obfuscated. Limited strains in specific-pathogen-free mice are capable of inducing tumorigenesis, and no strain yet has induced colon tumors in germ-free mice. In a human xenograft model, $F$ nucleatum carcinogenesis was associated with a bacterial community in both primary and metastatic tumors. ${ }^{11}$ These results suggest additional factor(s), such as host cell somatic mutation or other microbial virulence determinants and/or interactions, are required, providing opportunities for the discovery of new mechanisms of $F$ nucleatum tumor development.

Escherichia coli are common gut commensal bacteria. Certain strains contain a 50-kb hybrid polyketidenonribosomal peptide synthase operon $\left(\mathrm{pks}^{+} \mathrm{E}\right.$ coli) that produces the genotoxin colibactin. ${ }^{12}$ Multiple investigations have defined the ability of colibactin to cause DNA interstrand crosslinks, DNA double-strand breaks, chromosomal aberrances, and cell cycle arrest in human cells in vitro. ${ }^{13-15}$ This damage combined with intestinal inflammation has previously been shown to promote tumorigenesis in mice. ${ }^{16}$ Although a very labile molecule, colibactin results in the formation of specific DNA adducts with subsequent cytotoxicity and mutations. ${ }^{17}$ Pleguezuelos-Manzano et $\mathrm{al}^{12}$ recently identified a distinct mutational signal in clonal organoids after injection with $p k s^{+}$Ecoli that was not present in organoids injected with an isogenic $p k s^{-} E$ coli, providing evidence that the virulence factor of this procarcinogenic bacterium can modify CEC DNA, a requirement for emergence of CRC. This mutational signature was detected in up to $16 \%$ of human cancer genomes, predominantly in CRC. ${ }^{12}$ The impact of this mutational sequence remains uncertain, given, for example, the study's experimental design (repetitive exposure to $\mathrm{pks}^{+} \mathrm{E}$ coli without microbiome competition) and lack of direct linkage of the mutational signature, $p k s^{+} E$ coli colonization, and the development of CRC in single persons.

Bacteroides fragilis is another commensal bacterium that composes $1 \%$ to $2 \%$ of the human microbiota. ${ }^{18}$ Enterotoxigenic $B$ fragilis (ETBF) produces the zincdependent metalloprotease toxin, $B$ fragilis toxin (BFT), that causes colon inflammation and has been associated with diarrhea, inflammatory bowel disease, and CRC. ETBF induces carcinogenesis by activating host CEC STAT3 and NF- $\kappa$ B pathways while generating mucosal IL-17- and myeloid-dominant inflammation. ${ }^{19,20}$ BFT binds an unknown CEC receptor triggering E-cadherin cleavage, yielding enhanced barrier permeability, activation of CEC Wnt signaling, upregulation of c-Myc expression, and increased CEC proliferation. ${ }^{18}$ Recent work within our laboratory revealed that BFT rapidly increases chromatin accessibility and alters gene transcription, including downregulation of $M U C 2$, the major protein of colonic mucus, and upregulation of CEACAM6, which acts as a receptor for adherent-invasive E coli (many of which are $\left.p k s^{+}\right) .{ }^{21}$ Furthermore, ETBF-induced tumors contain marked genomic hypermethylation compared with spontaneous tumors in the $A p c^{\text {min/+ }}$ mouse model of CRC. ${ }^{22}$ The hypermethylated regions correspond to reduced expression of genes with known tumorsuppressive functions, such as Hoxa5, Polg, Runxl, and Lgr6. $^{22}$ Together, these data indicate the potential for ETBF to both prime and potentiate tumorigenesis in the colon.

The bacteria described so far have extensive experimental support for their involvement in CRC; however, the list of putative tumorigenic bacteria continues to expand. For decades, bloodstream infections with Streptococcus gallolyticus have been associated with CRC. Emerging mechanisms include recruitment of tumor-infiltrating lymphocytes and myeloid cells that generate an immune-suppressive microenvironment fostering neoplasia. ${ }^{23}$ Enterococcus faecalis induces ROS production and leukocyte adducts, resulting in procarcinogenic inflammation and CEC mutations. ${ }^{24}$ Peptostreptococcus stomatis and Peptostreptococcus anaerobius have been identified to have the potential to influence the tumor microenvironment through production of acidic metabolites and induction of ROS..$^{25,26}$ Moreover, in a meta-analysis of CRC 16S rRNA amplicon sequence data, a limited consortium of bacteria were consistently associated with human CRC: $B$ fragilis, F nucleatum, $P$ stomatis, Parvimonas micra, and Gemella morbillorum. ${ }^{27}$ Notably, all but $B$ fragilis are oral microbiome members, with a strong theme emerging of linkage between the oral microbiome and CRC pathogenesis. ${ }^{27}$ In addition, recent work has identified that periodontitis generates oral microbiome-reactive T-helper type 17 cells that are imprinted with gut tropism and migrate to the inflamed gut, resulting in activation by translocated oral microbiota and subsequent development of colitis. ${ }^{28}$ Further investigation into the composition of the microbiome of patients with CRC and subsequent experimental analyses are likely to continue to reveal key bacteria that function to initiate and/or promote CRC.

\section{Impact of the Microbiome Composition and} Structure on CRC

Although individual bacteria have the ability to influence CRC, the composition and structure of the microbiome as a whole have an equal and critical impact on CRC. The disruption of a healthy microbiome, termed 
"dysbiosis," can lead to immune activation through Toll-like receptor and NOD-like receptor signaling, resulting in low-level colon inflammation known to underpin all CRC. ${ }^{5}$ Furthermore, the microbiome produces myriad metabolites driven by community structure and host diet that directly interact with CECs. Short chain fatty acids, such as butyrate, are produced by fermenting bacteria after consumption of nondigestible carbohydrates. ${ }^{29}$ Butyrate functions as an antiinflammatory molecule through downregulation of proinflammatory cytokines, induction of apoptosis in CRC cell lines, regulation of gene expression through inhibiting histone deacetylases, and modulation of colonic regulatory $\mathrm{T}$ cells. ${ }^{30-32}$ Paradoxically, butyrate induced proliferation of harvested MSH2-deficient CECs in vitro, ${ }^{33}$ suggesting that tight control of this metabolite may be required to benefit the host and/ or that the host cell genotype modifies butyrate activity. The gut microbiome also converts bile acids from primary to secondary structures. ${ }^{34}$ Although secondary bile acids provide multiple benefits, including inhibition of Clostridioides difficile colonization, ${ }^{35}$ high abundance of these metabolites may induce oxidative DNA damage and promote colon tumor formation. ${ }^{36,37}$ Even the limited data available on the delicate balance of the metabolites produced by the gut microbiome lend importance to the overall microbial composition in CRC.

Colon biofilms, defined as highly dense communities of bacteria that invade the inner, dense, typically sterile mucus layer of the colon, provide resilient structures that allow continual interaction of bacterial communities with the host CECs. ${ }^{38}$ A recent study by Dejea et $\mathrm{al}^{39}$ determined that $53 \%$ of sporadic CRCs and surgical adenomas found in patients displayed colonic biofilms compared with the normal colon mucosa in $13 \%$ of healthy control subjects. Interestingly, $89 \%$ of the tumors containing biofilms were found on the right colon (up to the hepatic flexure), and all biofilms were associated with tumor-invasive bacteria. Notably, colon biofilms on histologically normal colon tissues modified CEC function, including an increase in STAT3 activation and loss of E-cadherin. ${ }^{39}$ Three microbiologic variants of colon biofilms were identified: polymicrobial (Bacteroidetes, Lachnospiraceae), polymicrobial with Fusobacteria blooms, and Proteobacteria dominant. ${ }^{27}$ In contrast, patients with FAP were found to have biofilms predominantly composed of ETBF and $p k s^{+} E$ coli, with these bacteria appearing to work coordinately to promote tumorigenesis. ${ }^{40}$ Namely, using the $A p c^{\mathrm{min} /+}$ mouse model, ETBF was identified to promote mucin degradation favoring the colonization of $\mathrm{pks}^{+}$E coli and culminating in a multifaceted CEC assault resulting in tumor formation. ${ }^{40}$ In additional work, human colon biofilms were procarcinogenic in murine models ${ }^{41}$ and may localize tumorigenic bacteria and virulence factor delivery directly to the colonic epithelium.

\section{Application of the Microbiome in CRC Prevention and Therapy}

The strong link between the microbiome and CRC presents an opportunity to develop screening modalities that could lead to targeted prevention and treatment of CRC. Current diagnosis of CRC relies on colonoscopy, guaiac fecal occult blood tests, fecal immunochemical tests, and/or multitarget stool DNA tests. ${ }^{1,42}$ Colonoscopy screening alone has resulted in a $90 \%$ chance of survival and declining CRC incidence rates among adults aged $>55$ years. ${ }^{43}$ However, the survival rate is reduced to $<10 \%$ for late diagnosis of metastatic CRC, ${ }^{1}$ demonstrating that early detection is key for patient outcomes. The goal is to use defined microbial signatures as a tool to lead to earlier and less invasive methods of CRC diagnosis. As yet, these aspirations have not been realized, but emerging data provide promise.

\section{Impact of the Microbiome on Therapy}

A growing body of evidence suggests the composition of the gut microbiome can promote or inhibit the anticancer effects of chemotherapeutic agents and immunotherapy. ${ }^{3}$ For instance, F nucleatum was shown to confer resistance to oxaliplatin and 5-FU through induction of autophagy. ${ }^{8}$ Microbial enzymes can modify chemotherapeutics, enhancing their harmful adverse effects, such as the reactivation of irinotecan through gut bacterial $\beta$-glucuronidase enzymes. ${ }^{44}$ Enrichment of bacteria containing vitamin B biosynthesis pathways and polyamine transport were associated with immune checkpoint inhibitor-associated colitis. ${ }^{45}$ Interestingly, the varied composition of the microbiome correlates with both positive and negative immunotherapeutic responses. The gut microbiome is proposed as important to the success of immunotherapy, with most data limited to immune checkpoint inhibitors targeting the PD- 1 axis and/or the CTLA-4 axis in melanoma. ${ }^{3}$ However, across studies, consistent gut microbiome communities have not been identified as providing a positive response to anti-PD-1 and/or anti-CTLA-4 therapy. ${ }^{3}$ Nonetheless, studies to define the interactions between the microbiome and therapeutic agents present the potential to enhance successful CRC treatment among cancer types. Establishing a potential core bacterial group that demarcates potential immunotherapy responders or nonresponders is an active area of investigation.

\section{Microbial Therapeutics}

The importance of the microbiome in CRC pathogenesis and its potential to influence therapeutic outcomes 
provides an unprecedented opportunity to develop microbial-targeted treatments. Microbiota manipulation is currently being approached from a multitude of directions. ${ }^{46}$ Specific dietary intervention and the use of prebiotics that putatively enrich beneficial bacteria are being studied to prevent CRC or improve therapy. ${ }^{5}$ Removal of deleterious bacteria through narrow-spectrum antibiotics, species-specific bacteriophages, or monoclonal antibody therapy seeks a highly specific approach to microbial modulation. ${ }^{3}$ Interestingly, a recent study observed paradoxical associations of oral antibiotic use with an increased risk of colon cancer but a reduced risk of rectal cancer, underscoring the need for further investigations of targeted antibiotic treatments. ${ }^{47}$ Furthermore, vaccines may provide an immunomodulatory benefit against the specific virulence factors involved in CRC, such as BFT of ETBF and FadA of $F$ nucleatum, or the bacteria producing other tumorigenic molecules. The administration of therapeutic molecules that function to negate or scavenge toxic molecules produced by the microbiome, such as ROS or DNA damage-inducing agents, might reduce the potential mutation frequency of CEC. ${ }^{48}$ Engineered or naturally occurring probiotic bacteria could function to outcompete harmful species through colonization displacement and niche exclusion and by producing therapeutic molecules in situ. ${ }^{46}$ The success of the complete replacement or restoration of a patient's microbiome in elimination of $C$ difficile infection through fecal microbiota transfer ${ }^{49}$ lends strong support to the notion that microbiota manipulation can modify biology and, at least, local colon disease. However, human fecal microbiota transfer that lacks quality control or well-defined microbial membership has led to patient infections with enteric bacteria and multiple drug-resistant bacteria with patient deaths. ${ }^{50}$ Nonetheless, investigations that yield defined microbial candidates that limit colon carcinogenesis or promote, for example, immunotherapeutic responses would be worthy of human clinical trials. Together, microbiota therapies are currently in their infancy. However, clinical trials are currently underway for CRC and multiple other diseases, suggesting that the first FDA-approved live microbial therapeutic is in the near future. ${ }^{46}$

\section{Early-Onset CRC and the Microbiome}

The decreasing or stabilizing trend of sporadic CRC incidence rates in wealthier countries is now being countered by a striking increase in early-onset CRC (EO-CRC; CRC diagnosed before age 50 years). EO-CRC has emerged since the 1970s in both wealthier countries and others. ${ }^{51}$ Moreover, approximately 1 in 10 new diagnoses of CRC is EO-CRC, with an estimated 50 new cases daily in the United States and 10 deaths. ${ }^{51}$ Notably, a patient's risk of CRC is commonly estimated by family history and age; yet, patients with EO-CRC often have no family history of disease or other obvious risk factors, such as a predisposing condition. ${ }^{51}$ Although limited EO-CRC cohort members have defined genetic factors, such as Lynch syndrome or FAP, most patients ( $65 \%-80 \%$, dependent on age) do not have a known genetic predisposition to CRC. ${ }^{51}$ Furthermore, EO-CRC has increased in incidence at a more rapid pace than can be attributed to changes in population genetics. This provides strong evidence that the development of EO-CRC is highly influenced by environmental risk determinants. ${ }^{51}$ Microbiome dysbiosis at an early age as a result of poor diet, increased antibiotic use, obesity, and/or other factors is proposed but not proven to lead to persistent, procarcinogenic dysbiosis and subsequent EO-CRC. For instance, childhood obesity has increased $>200 \%$ since the early 1960 s and is a risk factor for CRC and other diseases (eg, diabetes, metabolic syndrome) that influence CRC risk..$^{52}$ Despite this, many patients with EO-CRC are not obese and live a healthy lifestyle. ${ }^{51}$ Investigations into the composition of the microbiome of patients with EO-CRC are warranted to determine whether a distinct microbial signature can be identified. Furthermore, as we learn more about specific bacteria as CRC instigators, screens for prevention and novel therapies may contribute to increase survival of patients with EO-CRC. The mechanisms leading to increased prevalence of and risks for EO-CRC currently remain a mystery. However, the microbiome of these patients certainly is one potential risk factor to be elucidated.

\section{Conclusions}

With all types of cancer, survivability is directly correlated with the extent of disease progression at the time of detection. Further understanding of the microbiome's impact on initiating and/or promoting CRC will propel the field to a new generation of screening and therapeutic modalities. This brief review discussed how specific bacteria and the overall structure of the microbiome influence CRC progression through production of harmful molecules, modulation of the host immune system, and alterations to the host CEC genome. Use and additional pursuit of this knowledge should inform future patient care through the design of specific screens and microbial-based therapies, with the ultimate goal of improved patient outcomes. The critical importance of the microbiome in CRC provides a wellspring of research opportunities that we predict will one day revolutionize CRC standards of clinical practice.

Submitted November 3, 2020; final revision received May 6, 2021; accepted for publication May 19, 2021.

Disclosures: Dr. Knippel has disclosed having no financial interests arrangements, affiliations, or commercial interests with the manufacturers of 
any products discussed in this article or their competitors. Dr. Sears has disclosed receiving grant support from Bristol Myers Squibb and Janssen.

Funding: Research reported in this publication was supported by the $\mathrm{NIH}$ under award number R01 CA196845, Bloomberg Philanthropies (C.L. Sears), and the Cancer Research UK's Grand Challenge Initiative C10674/A27140 (C.L. Sears).
Disclaimer: The content is solely the responsibility of the authors and does not necessarily represent the official views of the $\mathrm{NIH}$.

Correspondence: Cynthia L. Sears, MD, Johns Hopkins University School of Medicine, 1550 Orleans Street, CRB2 Building, Suite 1M.05, Baltimore, MD 21231. Email: csears@jhmi.edu

\section{References}

1. Keum N, Giovannucci E. Global burden of colorectal cancer: emerging trends, risk factors and prevention strategies. Nat Rev Gastroenterol Hepatol 2019;16:713-732.

2. Allen J, Sears CL. Impact of the gut microbiome on the genome and epigenome of colon epithelial cells: contributions to colorectal cancer development. Genome Med 2019;11:11.

3. Wong SH, Yu J. Gut microbiota in colorectal cancer: mechanisms of action and clinical applications. Nat Rev Gastroenterol Hepatol 2019; 16:690-704.

4. Golofast B, Vales K. The connection between microbiome and schizophrenia. Neurosci Biobehav Rev 2020;108:712-731.

5. Tilg H, Adolph TE, Gerner RR, et al. The intestinal microbiota in colorectal cancer. Cancer Cell 2018;33:954-964.

6. Sender R, Fuchs S, Milo R. Revised estimates for the number of human and bacteria cells in the body. PLoS Biol 2016;14:e1002533.

7. Ternes D, Karta J, Tsenkova M, et al. Microbiome in colorectal cancer how to get from meta-omics to mechanism? Trends Microbiol 2020; 28:401-423.

8. Brennan CA, Garrett WS. Fusobacterium nucleatum - symbiont, opportunist and oncobacterium. Nat Rev Microbiol 2019 ; 17:156-166.

9. Rubinstein MR, Wang $X$, Liu W, et al. Fusobacterium nucleatum promotes colorectal carcinogenesis by modulating $\mathrm{E}$-cadherin/ $\beta$-catenin signaling via its FadA adhesin. Cell Host Microbe 2013;14:195-206.

10. Gur C, Ibrahim Y, Isaacson B, et al. Binding of the Fap2 protein of Fusobacterium nucleatum to human inhibitory receptor TIGIT protects tumors from immune cell attack. Immunity 2015;42:344-355.

11. Bullman S, Pedamallu CS, Sicinska E, et al. Analysis of Fusobacterium persistence and antibiotic response in colorectal cancer. Science 2017;358: 1443-1448.

12. Pleguezuelos-Manzano C, Puschhof J, Rosendahl Huber A, et al. Mutational signature in colorectal cancer caused by genotoxic pks $+\mathrm{E}$. coli. Nature 2020;580:269-273.

13. Nougayrède JP, Homburg S, Taieb F, et al. Escherichia coli induces DNA double-strand breaks in eukaryotic cells. Science $2006 ; 313$ : 848-851.

14. Bossuet-Greif N, Vignard J, Taieb F, et al. The colibactin genotoxin generates DNA interstrand cross-links in infected cells. MBio 2018;9: e02393-17

15. Zhang Z, Aung KM, Uhlin BE, et al. Reversible senescence of human colon cancer cells after blockage of mitosis/cytokinesis caused by the CNF1 cyclomodulin from Escherichia coli. Sci Rep 2018;8:17780.

16. Arthur JC, Perez-Chanona E, Mühlbauer M, et al. Intestinal inflammation targets cancer-inducing activity of the microbiota. Science 2012;338:120-123.

17. Wilson MR, Jiang Y, Villalta PW, et al. The human gut bacterial genotoxin colibactin alkylates DNA. Science 2019;363:eaar7785.

18. Sears $C L$, Geis AL, Housseau F. Bacteroides fragilis subverts mucosal biology: from symbiont to colon carcinogenesis. J Clin Invest 2014;124:4166-4172.

19. Thiele Orberg E, Fan H, Tam AJ, et al. The myeloid immune signature of enterotoxigenic Bacteroides fragilis-induced murine colon tumorigenesis. Mucosal Immunol 2017;10:421-433.

20. Chung $L$, Orberg ET, Geis AL, et al. Bacteroides fragilis toxin coordinates a pro-carcinogenic inflammatory cascade via targeting of colonic epithelial cells. Cell Host Microbe 2018;23:421.

21. Allen J, Hao S, Sears CL, et al. Epigenetic changes induced by Bacteroides fragilis toxin. Infect Immun 2019;87:e00447-18.

22. Maiuri $A R$, Peng $M$, Podicheti $R$, et al. Mismatch repair proteins initiate epigenetic alterations during inflammation-driven tumorigenesis. Cancer Res 2017;77:3467-3478.

23. Zhang Y, Weng Y, Gan H, et al. Streptococcus gallolyticus conspires myeloid cells to promote tumorigenesis of inflammatory bowel disease. Biochem Biophys Res Commun 2018;506:907-911.
24. Wang $X$, Yang $Y$, Huycke MM. Commensal bacteria drive endogenous transformation and tumour stem cell marker expression through a bystander effect. Gut 2015;64:459-468.

25. Purcell RV, Visnovska M, Biggs PJ, et al. Distinct gut microbiome patterns associate with consensus molecular subtypes of colorectal cancer. Sci Rep 2017:7:11590.

26. Tsoi H, Chu ESH, Zhang $X$, et al. Peptostreptococcus anaerobius induces intracellular cholesterol biosynthesis in colon cells to induce proliferation and causes dysplasia in mice. Gastroenterology 2017;152: 1419-1433.e5.

27. Drewes JL, White JR, Dejea CM, et al. High-resolution bacterial $16 \mathrm{~S}$ rRNA gene profile meta-analysis and biofilm status reveal common colorectal cancer consortia. NPJ Biofilms Microbiomes 2017;3:34.

28. Kitamoto S, Nagao-Kitamoto H, Jiao Y, et al. The intermucosal connection between the mouth and gut in commensal pathobiont-driven colitis. Cell 2020;182:447-462.e14.

29. Vital M, Howe AC, Tiedje JM. Revealing the bacterial butyrate synthesis pathways by analyzing (meta)genomic data. MBio 2014;5: e00889-14

30. Singh N, Gurav A, Sivaprakasam S, et al. Activation of Gpr109a, receptor for niacin and the commensal metabolite butyrate, suppresses colonic inflammation and carcinogenesis. Immunity 2014;40: 128-139.

31. Furusawa $Y$, Obata $Y$, Fukuda S, et al. Commensal microbe-derived butyrate induces the differentiation of colonic regulatory T cells. Nature 2013; 504:446-450.

32. Smith PM, Howitt MR, Panikov N, et al. The microbial metabolites, shortchain fatty acids, regulate colonic Treg cell homeostasis. Science 2013;341: 569-573.

33. Belcheva A, Irrazabal T, Robertson SJ, et al. Gut microbial metabolism drives transformation of MSH2-deficient colon epithelial cells. Cell 2014; 158:288-299.

34. Ridlon JM, Harris SC, Bhowmik S, et al. Consequences of bile salt biotransformations by intestinal bacteria. Gut Microbes 2016;7: 22-39.

35. Britton RA, Young VB. Role of the intestinal microbiota in resistance to colonization by Clostridium difficile. Gastroenterology 2014;146:15471553.

36. Bernstein $\mathrm{H}$, Bernstein $\mathrm{C}$, Payne $\mathrm{CM}$, et al. Bile acids as endogenous etiologic agents in gastrointestinal cancer. World J Gastroenterol 2009;15: 3329-3340.

37. Bernstein $\mathrm{C}$, Holubec $\mathrm{H}$, Bhattacharyya AK, et al. Carcinogenicity of deoxycholate, a secondary bile acid. Arch Toxicol 2011;85:863-871.

38. Domingue JC, Drewes JL, Merlo CA, et al. Host responses to mucosal biofilms in the lung and gut. Mucosal Immunol 2020;13: 413-422.

39. Dejea CM, Wick EC, Hechenbleikner EM, et al. Microbiota organization is a distinct feature of proximal colorectal cancers. Proc Natl Acad Sci USA 2014:111:18321-18326.

40. Dejea CM, Fathi P, Craig JM, et al. Patients with familial adenomatous polyposis harbor colonic biofilms containing tumorigenic bacteria. Science 2018;359:592-597.

41. Tomkovich S, Dejea CM, Winglee K, et al. Human colon mucosal biofilms from healthy or colon cancer hosts are carcinogenic. J Clin Invest 2019;129: 1699-1712.

42. Imperiale TF, Ransohoff DF, Itzkowitz SH. Multitarget stool DNA testing for colorectal-cancer screening. N Engl J Med 2014;370:1287-1297.

43. Bjerrum A, Milter MC, Andersen $\mathrm{O}$, et al. Risk stratification and detection of new colorectal neoplasms after colorectal cancer screening with faecal occult blood test: experiences from a Danish screening cohort. Eur J Gastroenterol Hepatol 2015;27:1433-1437.

44. Wallace BD, Roberts $A B$, Pollet RM, et al. Structure and inhibition of microbiome $\beta$-glucuronidases essential to the alleviation of cancer drug toxicity. Chem Biol 2015;22:1238-1249. 
45. Dubin K, Callahan MK, Ren B, et al. Intestinal microbiome analyses identify melanoma patients at risk for checkpoint-blockade-induced colitis. Nat Commun 2016;7:10391.

46. Jimenez M, Langer R, Traverso G. Microbial therapeutics: new opportunities for drug delivery. J Exp Med 2019;216:1005-1009.

47. Zhang J, Haines C, Watson AJM, et al. Oral antibiotic use and risk of colorectal cancer in the United Kingdom, 1989-2012: a matched casecontrol study. Gut 2019;68:1971-1978

48. Irrazabal T, Thakur BK, Kang M, et al. Limiting oxidative DNA damage reduces microbe-induced colitis-associated colorectal cancer. Nat Commun 2020;11:1802.
49. Cammarota G, laniro G, Gasbarrini A. Fecal microbiota transplantation for the treatment of Clostridium difficile infection: a systematic review. J Clin Gastroenterol 2014;48:693-702.

50. DeFilipp Z, Bloom PP, Torres Soto $\mathrm{M}$, et al. Drug-resistant E. coli bacteremia transmitted by fecal microbiota transplant. N Engl J Med 2019;381:2043-2050.

51. Stoffel EM, Murphy CC. Epidemiology and mechanisms of the increasing incidence of colon and rectal cancers in young adults. Gastroenterology 2020;158:341-353.

52. Ogden $\mathrm{CL}$, Carroll MD, Lawman HG, et al. Trends in obesity prevalence among children and adolescents in the United States, 1988-1994 through 2013-2014. JAMA 2016:315:2292-2299.
2021

NCCN Virtual Nursing Forum:

Advancing Oncology Nursing in Hematologic Malignancies
October 8, 2021 10:30 AM - 3:30 PM EDT

NCCN.org/hem-nursing 